\title{
Correction to: Short-Term Symptomatic Relief in Gastroesophageal Reflux Disease: A Comparative Study of Esomeprazole and Vonoprazan
}

\author{
Kouichi Sakurai ${ }^{1} \cdot$ Hiroko Suda $^{1} \cdot$ Satomi Fujie ${ }^{1} \cdot$ Takayuki Takeichi $^{1} \cdot$ Ayako Okuda $^{1} \cdot$ Tetsuya Murao $^{1}$. \\ Kiwamu Hasuda ${ }^{1} \cdot$ Masahiro Hirano $^{2} \cdot$ Kiyoharu Ito $^{3} \cdot$ Katsuie Tsuruta $^{4} \cdot$ Masahiro Hattori $^{1}$
}

Published online: 28 February 2019

(c) The Author(s) 2019

\section{Correction to: \\ Digestive Diseases and Sciences (2019) 64:815-822 \\ https://doi.org/10.1007/s10620-018-5365-0}

The original version of the article unfortunately contained percentage errors in second and third paragraphs of GerdQ Score section. Below is the corrected version.

We performed a stratified analysis of patients with NERD and erosive esophagitis. The proportions of patients with sufficient relief in NERD were $38.5,84.6$, and $84.6 \%$ of 13 patients in the esomeprazole group and 22.2, 66.7, and $77.8 \%$ of 9 patients in the vonoprazan group at 1,2 , and 4 weeks, respectively. The proportions of patients with complete resolution in NERD were 23.1, 53.8, and $69.2 \%$ in the esomeprazole group and $22.2,22.2$, and $33.3 \%$ in the vonoprazan group at 1,2 , and 4 weeks, respectively. There were no significant differences in the rates of sufficient relief and

The original article can be found online at https://doi.org/10.1007/ s10620-018-5365-0.

\author{
Kouichi Sakurai \\ sakuraiko@jcom.zaq.ne.jp \\ Hiroko Suda \\ sudahiro0825@gmail.com \\ Satomi Fujie \\ sa-fu@hattori-clinic.com \\ Takayuki Takeichi \\ ta-ta@hattori-clinic.com \\ Ayako Okuda \\ a-oku@hattori-clinic.com \\ Tetsuya Murao \\ te-mura@hattori-clinic.com \\ Kiwamu Hasuda \\ ki-hasu@hattori-clinic.com \\ Masahiro Hirano \\ gihirano@gaea.ocn.ne.jp
}

complete resolution in NERD at 1,2, or 4 weeks between the esomeprazole group and the vonoprazan group.

The proportions of patients with sufficient relief in erosive esophagitis were $66.7,91.7$, and $91.7 \%$ of 12 patients in the esomeprazole group and $69.2,76.9$, and $84.6 \%$ of 13 patients in the vonoprazan group at 1,2 , and 4 weeks, respectively. The proportions of patients with complete resolution in erosive esophagitis were $33.3,41.7$, and $58.3 \%$ in the esomeprazole group and 46.2, 46.2, and $61.5 \%$ in the vonoprazan group at 1, 2, and 4 weeks, respectively. There were no significant differences in the rates of sufficient relief and complete resolution at 1,2 , or 4 weeks between the esomeprazole group and the vonoprazan group in erosive esophagitis.

Kiyoharu Ito

itoh@ozakiiin.or.jp

Katsuie Tsuruta

tsurukatsu@gmail.com

Masahiro Hattori

m-hattori@hattori-clinic.com

1 Hattori Clinic, 2-12-35 Shin-machi, Chuo-ku, Kumamoto City, Kumamoto 860-0004, Japan

2 Hirano Gastroenterology Clinic, 2-3029-2, Oonuki-machi, Nobeoka City, Miyazaki 882-0803, Japan

3 Ozaki Clinic, 1-8 Hon-machi, Uto City, Kumamoto 869-0431, Japan

4 Tsuruta Hospital, 10-112 Hotakubohon-machi, Higashi-ku, Kumamoto City, Kumamoto 862-0925, Japan 